\title{
Molecular subtyping and genomic profiling expand precision medicine in refractory metastatic triple-negative breast cancer:
} the FUTURE trial

Yi-Zhou Jiang ${ }^{1,2}$, Yin Liu ${ }^{1,2}$, Yi Xiao ${ }^{1,2}$, Xin Hu$^{1,2}$, Lin Jiang ${ }^{1,2}$, Wen-Jia Zuo ${ }^{1,2}$, Ding Ma ${ }^{1,2}$, Jiahan Ding ${ }^{1,2}$, Xiaoyu Zhu $^{3}$, Jianjun Zou ${ }^{3}$, Claire Verschraegen ${ }^{4}$, Daniel G. Stover ${ }^{5}$, Virginia Kaklamani ${ }^{6}$, Zhong-Hua Wang ${ }^{1,2}$ and Zhi-Ming Shao ${ }^{1,2}$

Triple-negative breast cancer (TNBC) is a highly heterogeneous disease, and molecular subtyping may result in improved diagnostic precision and targeted therapies. Our previous study classified TNBCs into four subtypes with putative therapeutic targets. Here, we conducted the FUTURE trial (ClinicalTrials.gov identifier: NCT03805399), a phase Ib/ll subtyping-based and genomic biomarkerguided umbrella trial, to evaluate the efficacy of these targets. Patients with refractory metastatic TNBC were enrolled and stratified by TNBC subtypes and genomic biomarkers, and assigned to one of these seven arms: (A) pyrotinib with capecitabine, (B) androgen receptor inhibitor with CDK4/6 inhibitor, (C) anti PD-1 with nab-paclitaxel, (D) PARP inhibitor included, (E) and (F) anti-VEGFR included, or (G) mTOR inhibitor with nab-paclitaxel. The primary end point was the objective response rate (ORR). We enrolled 69 refractory metastatic TNBC patients with a median of three previous lines of therapy (range, 1-8). Objective response was achieved in $20(29.0 \%, 95 \%$ confidence interval $(\mathrm{Cl}): 18.7 \%-41.2 \%)$ of the 69 intention-to-treat (ITT) patients. Our results showed that immunotherapy (arm C), in particular, achieved the highest ORR (52.6\%, 95\% Cl: $28.9 \%-75.6 \%)$ in the ITT population. Arm $\mathrm{E}$ demonstrated favorable ORR ( $26.1 \%, 95 \% \mathrm{Cl}: 10.2 \%-48.4 \%$ in the ITT population) but with more high grade ( $\geq 3)$ adverse events. Somatic mutations of TOP2A and CD8 immunohistochemical score may have the potential to predict immunotherapy response in the immunomodulatory subtype of TNBC. In conclusion, the phase Ib/II FUTURE trial suggested a new concept for TNBC treatment, demonstrating the clinical benefit of subtyping-based targeted therapy for refractory metastatic TNBC.

Cell Research (2021) 31:178-186; https://doi.org/10.1038/s41422-020-0375-9

\section{INTRODUCTION}

Triple-negative breast cancer (TNBC) encompasses a subset of breast cancers that lack expression of the estrogen receptor (ER), progesterone receptor (PR), and human epidermal growth factor receptor 2 (HER2). ${ }^{1,2}$ TNBCs account for 10 to $20 \%$ of newly diagnosed breast cancer cases and are associated with higher incidence of visceral metastases, higher risk of early recurrence and worse prognosis. ${ }^{3,4}$

In recent years, the consensus is that TNBC is a highly heterogeneous disease, ${ }^{5-8}$ and this may have implication for TNBC treatment choice. Our previous study presented a multiomic profiling of 465 Chinese patients with TNBCs and provided the largest genomically characterized TNBC dataset to date. ${ }^{8}$ We classified TNBCs into four mRNA subtypes with distinct molecular features: (1) luminal androgen receptor (LAR), (2) immunomodulatory (IM), (3) basal-like immune-suppressed (BLIS), and (4) mesenchymal-like (MES), identified the genomic aberrations that drive each TNBC mRNA subtype, and provided additional insights into TNBC heterogeneity and potential therapeutic options.
Advancement in genomics has fueled the efforts toward "precision oncology", targeting cancers on the basis of their genetic mutations. Clinical trials focusing on precision oncology are often classified as "umbrella trials", "platform trials" and "basket trials". Umbrella trials evaluate multiple targeted therapies for one single disease, such as BATTLE-2 study and Lung-MAP study. ${ }^{9-13}$ However, these studies mainly focused on genomic targets, and did not take molecular subtyping into consideration in the study design. Platform trials set a platform to evaluate multiple targeted therapies for multiple diseases, mainly focusing on refractory solid tumors and rare tumors, such as IMPACT, I-PREDICT and WINTHER platforms. ${ }^{14-16}$ Basket trials refer to designs in which one targeted therapy is evaluated on multiple diseases that have common genetic aberrations. Most precision medicine trials focus on DNA abnormalities, but only a few tumors have tractable genomic alterations. There is an urgent need to explore therapeutic targets beyond the identification of genomic driver aberrations.

Although previous clinical trials have studied several targeted therapies for TNBC, most of these trials were single- or double-arm,

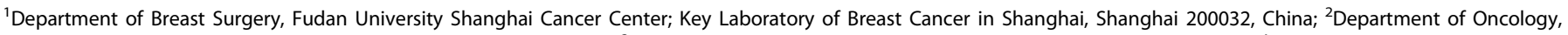

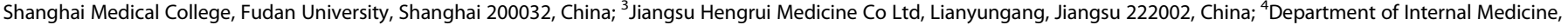

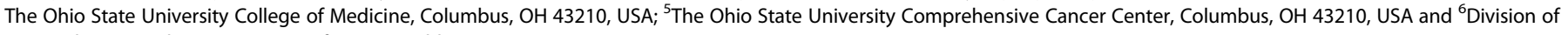
Hematology/Oncology, University of Texas Health Science Center San Antonio, San Antonio, TX 78284, USA

Correspondence: Zhong-Hua Wang (zhonghuawang95@hotmail.com) or Zhi-Ming Shao (zhimingshao@yahoo.com)

These authors contributed equally: Yi-Zhou Jiang, Yin Liu, Yi Xiao

Received: 19 April 2020 Accepted: 30 June 2020

Published online: 27 July 2020 


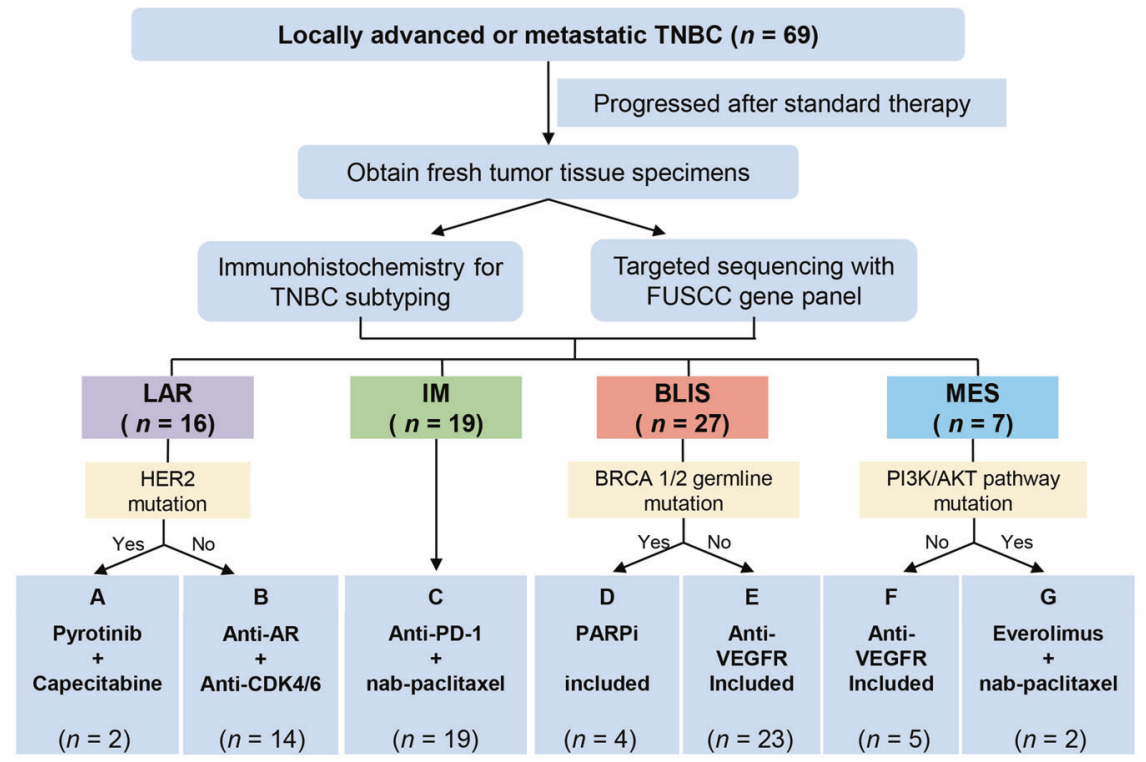

Fig. 1 The FUTURE trial schema: integrating TNBC subtyping and genomic targeting. $n$, number of the patients; TNBC, triple-negative breast cancer; FUSCC, Fudan University Shanghai Cancer Center; LAR, luminal androgen receptor; IM, immunomodulatory; BLIS, basal-like immune-suppressed; MES, mesenchymal-like; AR, androgen receptor; PD-1, programmed cell death-1; PARPi, poly ADP-ribose polymerase inhibitor; VEGFR, vascular endothelial growth factor receptor.

and they did not subtype TNBC for a specific target, which may limit the treatment efficacy. ${ }^{17-21}$ Referring to previous umbrella trials, we now present a phase Ib/ll Fudan University Shanghai Cancer Center TNBC umbrella (FUTURE) trial, which for the first time combined the TNBC subtyping and genomic sequencing-guided targeted therapy for refractory metastatic TNBC patients (Fig. 1). The FUTURE trial first simplified the TNBC mRNA subtypes by an immunohistochemical (IHC) method using three representative markers. Combining with TNBC IHC subtype-specific genomic features, the FUTURE trial allows most of the patients to enter the corresponding precision treatment arm. We aimed to evaluate the efficacy and safety of multiple precision treatments in heavily pretreated patients with refractory metastatic TNBC who had received a median of three previous lines of therapy. For each enrolled patient, one recurrent or metastatic tumor site was prospectively biopsied for IHC subtyping and targeted sequencing (Supplementary information, Fig. S1 and Table S1). Based on the identified TNBC IHC subtype and genomic biomarkers, patients were then assigned to a certain arm of the study. Here, we report the interim analysis of the FUTURE trial.

\section{RESULTS}

Patient characteristics

Between October 18th, 2018 and March 25th, 2020, 69 patients were enrolled in the FUTURE trial, out of 87 screened patients (Fig. 1 and Supplementary information, Fig. S2). Table 1 summarizes the clinical characteristics of the 69 enrolled patients. These patients were heavily pretreated (median of three previous antitumor regimens in the metastatic setting (range, 1-8)), most of whom received taxanes (99\%), anthracyclines (86\%), platinums $(88 \%)$, vinorelbine $(81 \%)$, capecitabine $(75 \%)$, and gemcitabine (72\%) before enrollment. Other characteristics were as follows: $81 \%$ had two or more metastatic organs, and $67 \%$ experienced disease progression within 6 months of their first-line chemotherapy (Table 1), reflecting the heavy tumor burden and resistant disease of the enrolled patients. Detailed patient information is listed in Supplementary information, Table S2.

The median duration of follow-up at the time of cutoff (April 7th, 2020) was 9.2 months (interquartile range (IQR)
5.3-12.2 months). At the data cutoff time, 9 (13.0\%) of 69 patients remained on treatment. $40(66.7 \%)$ of the 60 patients discontinued the study due to disease progression. Three (5.0\%) patients discontinued the study as a result of serious adverse events. Fourteen $(23.3 \%)$ patients withdrew from the study. In addition, two $(3.3 \%)$ patients were lost to follow-up before the first post-baseline tumor assessment, and one (1.7\%) patient had a ruptured chest wall lesion as her only site of measurable disease but could not be measured with imaging examination (Supplementary information, Fig. S2).

\section{Efficacy}

Patients were enrolled into the following arms based on their TNBC subtypes and genomic features: (A) pyrotinib with capecitabine, (B) androgen receptor inhibitor with CDK4/6 inhibitor, (C) anti PD-1 with nab-paclitaxel, (D) PARP inhibitor included, (E) and (F) anti-VEGFR included, or (G) mTOR inhibitor with nab-paclitaxel. Treatment efficacy of each arm is illustrated in Fig. 2a. Detailed imaging information from representative samples are illustrated in Supplementary information, Fig. S3. Fifty of the 69 enrolled patients underwent at least one post-baseline assessment. In general, objective response (complete response $(\mathrm{CR})+$ partial response (PR)) was achieved in $20(29.0 \%, 95 \% \mathrm{Cl}$ : $18.7 \%-41.2 \%$ ) of 69 intention-to-treat (ITT) patients and in 20 (40.0\%, 95\% Cl: $26.4 \%-54.8 \%)$ of 50 per-protocol (PP) patients. Disease control (CR + PR + stable disease (SD)) was achieved in $29(42.0 \%, 95 \% \mathrm{Cl}: 30.2 \%-54.5 \%$, in ITT population; $58.0 \%, 95 \% \mathrm{Cl}$ : $43.2 \%-71.8 \%$, in PP population, respectively) patients. In the 18 patients whose data were available, we compared the duration of treatment provided by the FUTURE trial with that of the patient's most recent anti-cancer treatment before enrollment. The median duration of treatment provided by FUTURE trial was 3.5 months, whereas that of previous anti-cancer treatment was 2.4 months $(P=0.02$, Fig. $2 b)$.

Nineteen patients enrolled in arm $C$ were assigned to the treatment of immune checkpoint inhibitor (ICI) plus nab-paclitaxel, with sixteen $(84.2 \%)$ patients undergoing at least one post-baseline assessment, one (5.3\%) patient was in poor physical condition, and was unable to travel to the hospital for assessment, one (5.3\%) patient was lost to follow-up, and one (5.3\%) patient had a ruptured 
Table 1. Baseline characteristics of the enrolled patients.

\begin{tabular}{|c|c|}
\hline Characteristics & Patients $(n=69)$ \\
\hline \multicolumn{2}{|c|}{ Median age at enrollment - year (range) } \\
\hline & $51(28-74)$ \\
\hline \multicolumn{2}{|l|}{ ECOG - no. (\%) } \\
\hline 0 & $3(4 \%)$ \\
\hline 1 & $54(78 \%)$ \\
\hline 2 & $12(17 \%)$ \\
\hline \multicolumn{2}{|c|}{ Previous lines of treatment - median no. (range) } \\
\hline & $3(1-8)$ \\
\hline \multicolumn{2}{|c|}{$\begin{array}{l}\text { Previous use of taxanes or anthracyclines for metastatic } \\
\text { or nonmetastatic disease }- \text { no. (\%) }\end{array}$} \\
\hline Taxanes & $68(99 \%)$ \\
\hline Anthracyclines & 59 (86\%) \\
\hline \multicolumn{2}{|c|}{$\begin{array}{l}\text { Previous use of chemotherapy drugs for } \\
\text { metastatic disease }- \text { no. (\%) }\end{array}$} \\
\hline Platinum agents & $61(88 \%)$ \\
\hline Gemcitabine & $50(72 \%)$ \\
\hline Capecitabine & $52(75 \%)$ \\
\hline Vinorelbine & $56(81 \%)$ \\
\hline Others & $15(22 \%)$ \\
\hline \multicolumn{2}{|c|}{ No. of metastatic organ - no. (\%) } \\
\hline 1 & $13(19 \%)$ \\
\hline 2 & $26(38 \%)$ \\
\hline $3+$ & $30(43 \%)$ \\
\hline \multicolumn{2}{|c|}{ Metastatic site - no. (\%) } \\
\hline Lymph nodes & $43(62 \%)$ \\
\hline Lung & $35(51 \%)$ \\
\hline Liver & $21(30 \%)$ \\
\hline Bone & $30(43 \%)$ \\
\hline Chest & $22(32 \%)$ \\
\hline Breast & $15(22 \%)$ \\
\hline Others & $14(20 \%)$ \\
\hline \multicolumn{2}{|c|}{ Progression-free interval of the first-line therapy (months) - no. (\%) } \\
\hline$<3$ & $24(35 \%)$ \\
\hline $3-6$ & $22(32 \%)$ \\
\hline$>6$ & $8(12 \%)$ \\
\hline Unknown & $15(22 \%)$ \\
\hline
\end{tabular}

chest wall lesion that could not be measured with imaging examination (Supplementary information, Fig. S2). Fig. 2c shows the waterfall plot of the sixteen evaluable patients with refractory metastatic TNBC in arm C. Ten (62.5\%) of the sixteen patients experienced a PR at first post-baseline evaluation. The objective response rate (ORR) of arm C was $52.6 \%$ (95\% Cl: $28.9 \%-75.6 \%)$ in the ITT population and $62.5 \%(95 \% \mathrm{Cl}: 35.4 \%-84.8 \%)$ in the PP population. Of the ten PR patients, median duration of response was 3.1 months (range 1.0-9.1 months; Fig. 2d, e). At the time of data cutoff (April 7th, 2020), six (60.0\%) of the ten PR patients had discontinued the treatment after disease progression. One $(10.0 \%)$ patient had discontinued the treatment as a result of serious adverse event (rupture of hemangioma of head and face). The remaining three PR patients are still being treated, and have thus far received anti PD-1 with nab-paclitaxel for 2.6-6.5 months (Fig. 2d, e).

Twenty-three patients were enrolled in arm E (anti-VEGFR), among whom seventeen (73.9\%) patients underwent at least one post-baseline assessment. The waterfall plot demonstrated that six patients in arm $E$ experienced objective response, with one CR and five PR (Fig. 2f). The ORR of arm E was $26.1 \%(95 \% \mathrm{Cl}$ : $10.2-48.4 \%)$ and $35.3 \%$ (95\% Cl: $14.2-61.7 \%)$ in the ITT and PP populations, respectively. Of the six patients with an objective response, the median duration of response was 4.1 months (range 1.1-9.8 months; Fig. $2 \mathrm{~g}$, h). Five (83.3\%) of six patients discontinued treatments after disease progression, one patient with supraclavicular lymph nodes metastasis after five lines of therapy experienced $C R$ after receiving apatinib for 6.6 months (Fig. 2g, h). Details regarding patients who did not undergo evaluation due to numerous reasons are listed in Supplementary information, Fig. S2.

\section{Toxicity}

Supplementary information, Table S3 summarizes the adverse events (AEs) of each arm. The most common treatment-related AEs of any grade were anemia $(n=46,67 \%)$, leukopenia $(n=41$, $59 \%)$, neutropenia $(n=30,43 \%)$, thrombocytopenia $(n=28,41 \%)$, fatigue $(n=26,38 \%)$, hypertension $(n=18,26 \%)$, proteinuria $(n=$ $18,26 \%)$, and elevated alanine aminotransferase (ALT) $(n=17$, 25\%) (Supplementary information, Table S3). Noticeably, except for hematologic high-grade $A E s$ ( $\geq$ grade three), most of the other high-grade AEs occurring in arm $E$ was related to apatinib (500 mg). High-grade AEs, including hypertension $(n=5,22 \%)$, proteinuria $(n=4,17 \%)$, hand-foot syndrome (HFS) $(n=4,17 \%)$, leukopenia $(n=2,9 \%)$, and elevated $\operatorname{ALT}(n=2,9 \%)$ were reported in at least two patients of arm E (Table 2). In particular, two patients whose best response were SD in arm E experienced severe HFS and elevated ALT, which resulted in the discontinuation of treatment before disease progression (Fig. $2 g$, h). Dose reduction occurred in six (40\%) of fifteen patients with apatinib monotherapy. In addition, one PR patient in arm $C$ discontinued the $\mathrm{ICl}$ therapy because of the rupture of hemangioma of head and face during the treatment, which was considered by the investigator to be drug-related.

Genomic landscape of refractory TNBC

In order to describe the genomic landscape of refractory TNBC, we conducted Fudan University Shanghai Cancer Center (FUSCC) next-generation sequencing (NGS) panel of targeted sequencing on the metastatic tumor samples (Supplementary information, Table S4). Most frequently mutated genes in refractory TNBC included TP53 (72\%), PIK3CA (18\%), PTEN (10\%), KMT2D (9\%) and TSC2 (9\%) (Fig. 3a). Metastatic TNBC generally exhibited a similar mutation landscape to their primary counterparts. A higher mutation frequency was observed in some infrequently mutated genes, such as PTPRD, TSC2, PLCG1, ARID1B, CREBBP and FAM47C (Fig. $3 \mathrm{~b}$ ). We also conducted a race comparison between our FUSCC cohort of Chinese metastatic TNBC and Memorial SloanKettering Cancer Center (MSKCC) cohort of American metastatic TNBC. ${ }^{22}$ A higher mutation frequency of TP53 was found in the MSKCC cohort (Fig. 3c). No other significant difference was found between the two populations.

Potential predictors of response in TNBC

We conducted clinical and genomic analysis to explore the potential predictors of response in refractory TNBC. Firstly, we compared the genomic difference between the patients whose best response was PR versus non-PR in arm C (immunotherapy for IM subtype). TOP2A mutation was found in two of the six non-PR patients of arm $C$, while no mutation was found in PR patients (Fig. 4a, b). One mutation appeared in the domain of DNA topoisomerase 2-like protein (PTZ00108) and the other appeared in the junction part between the two domains of TOP2A protein (Fig. 4C). We also compared the CD8 IHC score (defined as the number of CD8-positive cells divided by the total number of all types of cells on the pathological section of CD8 IHC staining, Supplementary Methods) between progressive disease (PD) and non-PD patients in arm C. Median CD8 score of non-PD patients was 30 while that of PD patients was $22.5(P=0.25$, Supplementary information, Fig. S4C). In addition, PD-L1 IHC score of the immune cells and tumor cells (defined as the number of PD-L1 positive immune cells divided by the total number of immune cells and the number of PD-L1 positive tumor cells divided by the 


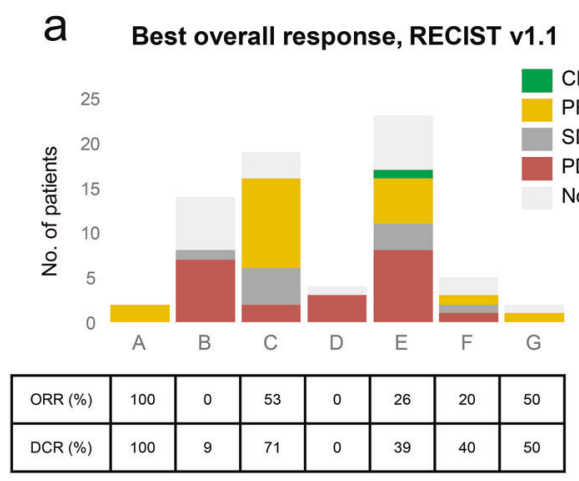

C

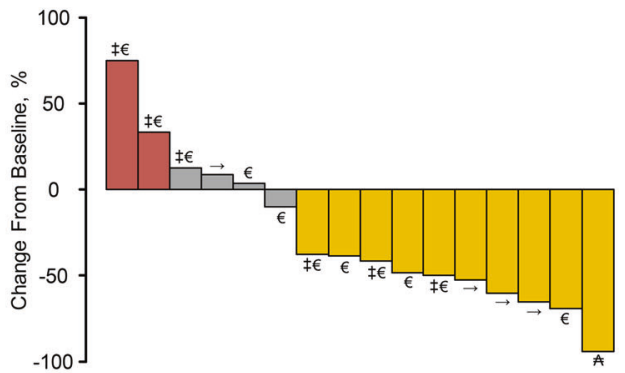

d

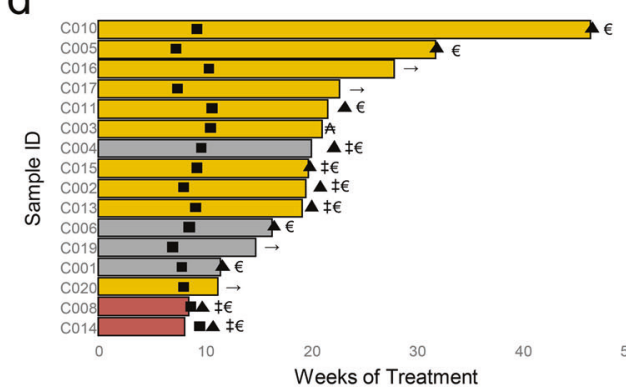

e

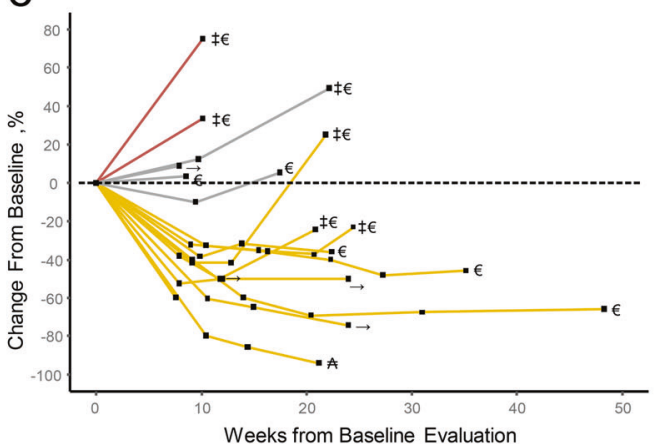

b

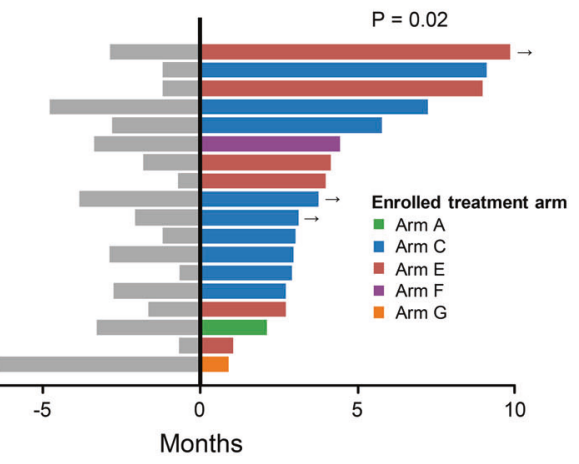

f

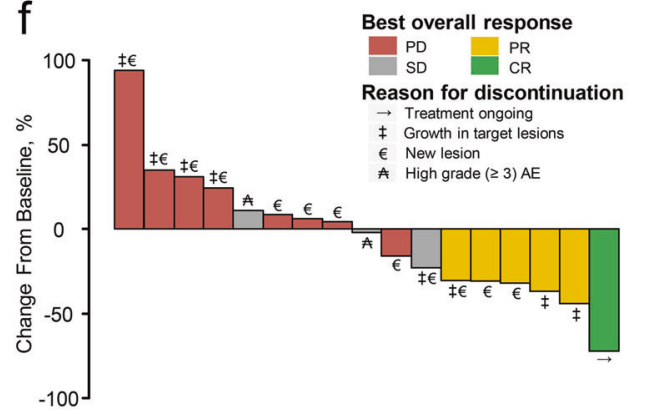

g

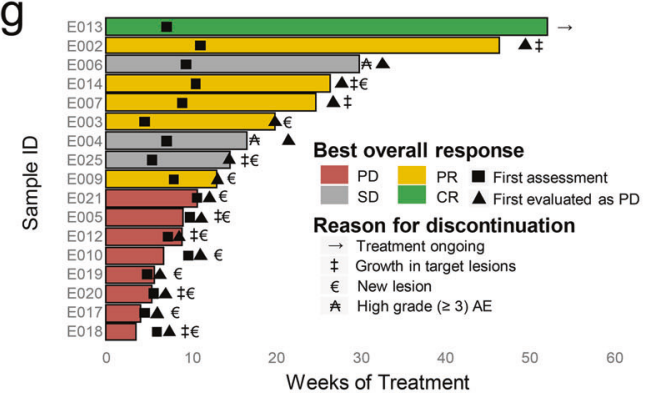

h

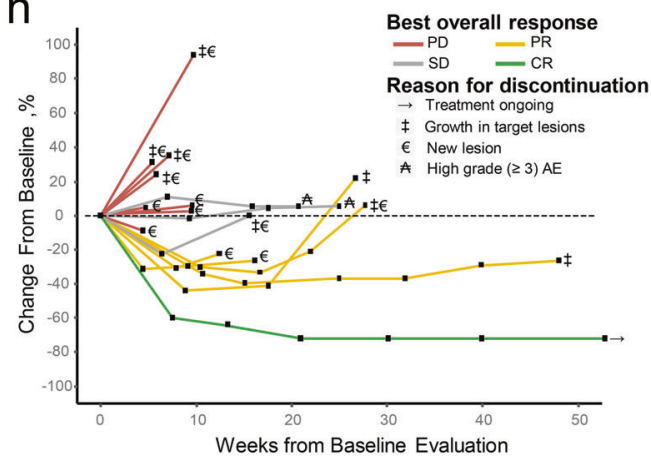

Fig. 2 Summary of therapy response. a Summary of the category of the best response in each arm of the FUTURE trial. $\mathbf{b}$ Duration of treatment in the FUTURE and of the last previous therapy in 18 patients in the PP population with available previous treatment duration information. $\mathbf{c}, \mathbf{f}$ Best percentage change from baseline in the sum of the longest diameters of target lesions in arm C (c) and arm E (f). $\mathbf{d}, \mathbf{g}$ Time to and durability of treatment in arm C (d) and arm E (g). e, h Longitudinal change from baseline in the sum of the longest diameters of target lesions in arm $C$ (e) and arm E (h). CR, complete response; PR, partial response; SD, stable disease; PD, progressive disease; AE, adverse event.

total number of tumor cells on the pathological section of PD-L1 IHC staining, respectively) were evaluated. Some PR patients illustrated higher PD-L1 IHC scores than non-PR patients (Supplementary information, Fig. S4c). Furthermore, we also conducted genomic and clinical analysis in the whole cohort and arm E. A tendency toward higher frequency of drug resistance-related mutations (such as PTEN, RB1 and NOTCH3) was observed in PD patients of the whole cohort and arm E (Supplementary information, Fig. S4a, b). There was a tendency of higher FOXC1 IHC score (defined as the number of positive tumor cells divided by the total number of tumor cells on the pathological section of FOXC1 IHC staining, Supplementary Methods) in SD plus PD patients compared with CR plus PR patients in arm $E(P=0.26$, Supplementary information, Fig. S4c). 
Table 2. Treatment-related adverse events in arm E.

\begin{tabular}{ll}
\hline Adverse event & No. (\%) \\
\hline Any grade occurring in at least two patients - no. (\%) & \\
Fatigue & $13(57 \%)$ \\
Anemia & $12(52 \%)$ \\
Thrombocytopenia & $11(48 \%)$ \\
Nausea & $6(26 \%)$ \\
Weight loss & $6(26 \%)$ \\
Cough & $3(13 \%)$ \\
Oral mucositis & $3(13 \%)$ \\
Neuropathy & $3(13 \%)$ \\
Vomiting & $2(9 \%)$ \\
Diarrhea & $2(9 \%)$ \\
Abdominal pain & $2(9 \%)$ \\
Grade 3-5 occurring in at least one patient - no. (\%) & \\
Hypertension (Grade 3) & $5(22 \%)$ \\
Proteinuria (Grade 3) & $4(17 \%)$ \\
HFS (Grade 3) & \\
Leukopenia (Grade 3) & $4(17 \%)$ \\
Elevated ALT (Grade 4) & $2(9 \%)$ \\
Neutropenia (Grade 4) & $2(9 \%)$ \\
Chest distress (Grade 3) & $1(4 \%)$ \\
Decreased appetite (Grade 3) & $1(4 \%)$ \\
\hline ALT, alanine aminotransferase; HFS, hand-foot syndrome. \\
${ }^{a}$ Shown are the adverse events that caused discontinuation of drug usage. \\
\hline
\end{tabular}

\section{DISCUSSION}

This phase Ib/II FUTURE trial confirmed the feasibility of a biopsymandated, subtyping-based and genomic biomarker-guided therapy in heavily pretreated refractory metastatic TNBCs. The trial indicated for the first time the potential role of TNBC subtyping and genomic testing in targeted therapy of refractory metastatic TNBCs. Furthermore, the FUTURE trial demonstrated favorable outcomes. The ORR and DCR of the 69 enrolled patients were $29.0 \%$ and $42.0 \%$, respectively. Specifically, arms $C$ and $\mathrm{E}$, where the $\mathrm{IM}$ and $B R C A 1 / 2$ gene wild type-BLIS subtypes were targeted with immunotherapy and anti-VEGFR therapy, respectively, accrued more patients and showed favorable outcomes.

A major focus of the FUTURE trial was to explore the efficacy of anti PD-1 with nab-paclitaxel for TNBCs of the IM subtype. Our previous study revealed that elevated immune cell signaling and tumor infiltrating lymphocytes were hallmarks of the IM subtype. High expression of immune checkpoint genes (such as PD-1, PDL1, and CTLA-4) suggested potential benefit from ICls., ${ }^{83}$ We applied this hypothesis to arm C of the FUTURE trial, and observed a durable efficacy of ICls for IM subtype TNBCs, with the ORR of $52.6 \%$ (95\% Cl: $28.9 \%-75.6 \%)$ in the ITT population and $62.5 \%$ (95\% Cl: $35.4 \%-84.8 \%$ ) in the PP population. Previous trials of later lines $\mathrm{ICl}$ monotherapy in metastatic TNBC demonstrated an ORR around $5 \%-10 \% .^{24-26}$ The ORR increased to around 20\% for the first-line monotherapy. ${ }^{17,27}$ Combination of ICl with chemotherapy was also extensively investigated. The KEYNOTE-150 phase lb/II study evaluated the efficacy of eribulin combined with pembrolizumab. ${ }^{28}$ The ORRs were $29.2 \%$ in first-line patients and $22 \%$ in second or later lines, respectively. The study initiated by Adams et al. evaluated the clinical efficacy of atezolizumab combined with nab-paclitaxel in metastatic TNBC patients. The ORR was $67 \%$ in the first-line, $25 \%$ in the second-line, and $29 \%$ in the third or later lines. ${ }^{29,30}$ A following first-line phase III study conducted in TNBCs, the IMpassion 130 trial, had a response rate of $56 \%$ and
$58.9 \%$ in the ITT population and in the PD-L1-positive subgroup, respectively. ${ }^{31}$ As mentioned above, the target population in our study was much more heavily pretreated, which meant these patients had progressed after using all accessible chemotherapies in the field of breast cancer, including anthracyclines, taxanes, cyclophosphamide, platinums, capecitabine, vinorelbine and gemcitabine. Compared with the trials listed above, the patients in the arm C of the FUTURE trial were enrolled after a median of three lines of therapy, and presented with a heavier disease burden, yet they still achieved a more favorable ORR (Supplementary information, Table S5). Hence, anti PD-1 could be a promising treatment for TNBC with IM subtype.

Furthermore, we revealed that TOP2A mutation and CD8 IHC score may be potential predictors of response in the immunotherapy treatment arm. TOP2A encodes the topoisomerase (DNA) II Alpha, relieving torsional stress by forming transient double strand breaks of DNA. ${ }^{32}$ Copy number variation and mRNA expression of $T O P 2 A$ were correlated with tumor progression and drug resistance of chemotherapy. ${ }^{33,34}$ However, the mutations of TOP2A were less explored. Our study revealed two mutations of TOP $2 A$ in non-PR patients of immunotherapy. The mutations of TOP $2 A$ might result in the change of junction peptides between functional domains and might influence the DNA binding of functional domains of TOP2A. Further, we revealed that CD8 IHC staining, which was used in the FUTURE study, might be a practical and feasible methodology for identifying TNBCs with IM subtypes and provide an indication for the administration of ICls.

Arm $\mathrm{E}$ explored the efficacy of VEGFR inhibitor in the treatment of refractory metastatic TNBCs of the BLIS subtype without BRCA1/ 2 germline mutation. The ORRs of arm $\mathrm{E}$ was $26.1 \%(95 \% \mathrm{Cl}$ : $10.2 \%-48.4 \%)$ and $35.3 \%(95 \% \mathrm{Cl}: 14.2 \%-61.7 \%)$ in the ITT and PP populations, respectively. A previous clinical trial reported a $10.7 \%$ ORR and $25.0 \%$ clinical benefit rate for apatinib treatment in heavily pretreated TNBC patients who did not undergo TNBC subtyping, ${ }^{35}$ and various clinical trials regarding bevacizumab have also shown similarly disappointing results. ${ }^{36,37}$ When TNBC patients of the BRCA1/2 wild-type BLIS subtype were targeted with anti-VEGFR therapy, their responses appeared to be more promising. However, it was noted that the response to anti-VEGFR therapy in these patients was heterogeneous, and was accompanied by significant toxicity from apatinib $(500 \mathrm{mg})$. Due to the frequency of high-grade $\mathrm{AEs}$, the researchers revised the treatment plan of arm E from apatinib $500 \mathrm{mg}$ to apatinib $250 \mathrm{mg}$ (or famitinib $20 \mathrm{mg}$ ) plus VP-16 $50 \mathrm{mg}$ (Supplementary Methods). The above two issues make us hesitant to perform future trials with apatinib, but encourage further study to uncover other druggable targets for BLIS subtype TNBCs without BRCA1/2 germline mutation.

Other arms also explored the drug efficacy based on proposed TNBC subtype classification. The LAR subtype was enriched with HER2 mutations (9\%), suggesting a possible benefit with the irreversible tyrosine kinase inhibitor pyrotinib. ${ }^{8}$ At the time of data cut-off, there were two assessable patients in arm A, both of whom achieved a PR after two cycles of therapy. Arms F and G enrolled five and two patients, with the ORRs of $20 \%$ and $50 \%$ in the ITT population, respectively. However, results from some of the treatment arms were inconsistent with our previous hypothesis. For example, our previous study discovered that LAR subtype TNBCs had less RB1 losses/deletions, and more frequent CDKN2A alterations, which may benefit from CDK4/6 inhibitors. ${ }^{8}$ However, only one of the eight assessable patients in arm B presented with SD at first evaluation, while the remaining seven patients progressed despite being treated with anti-AR combined with anti-CDK4/6 therapy. Interestingly, the genomic analysis showed that all TNBCs in arm B were CDKN2A neutral, which may decrease the efficacy of CDK4/6 inhibitors (Supplementary information, Fig. S5). Genomic landscape of TNBCs in arm B also suggested other potential targets, such as mutations in PI3K-AKT-mTOR 


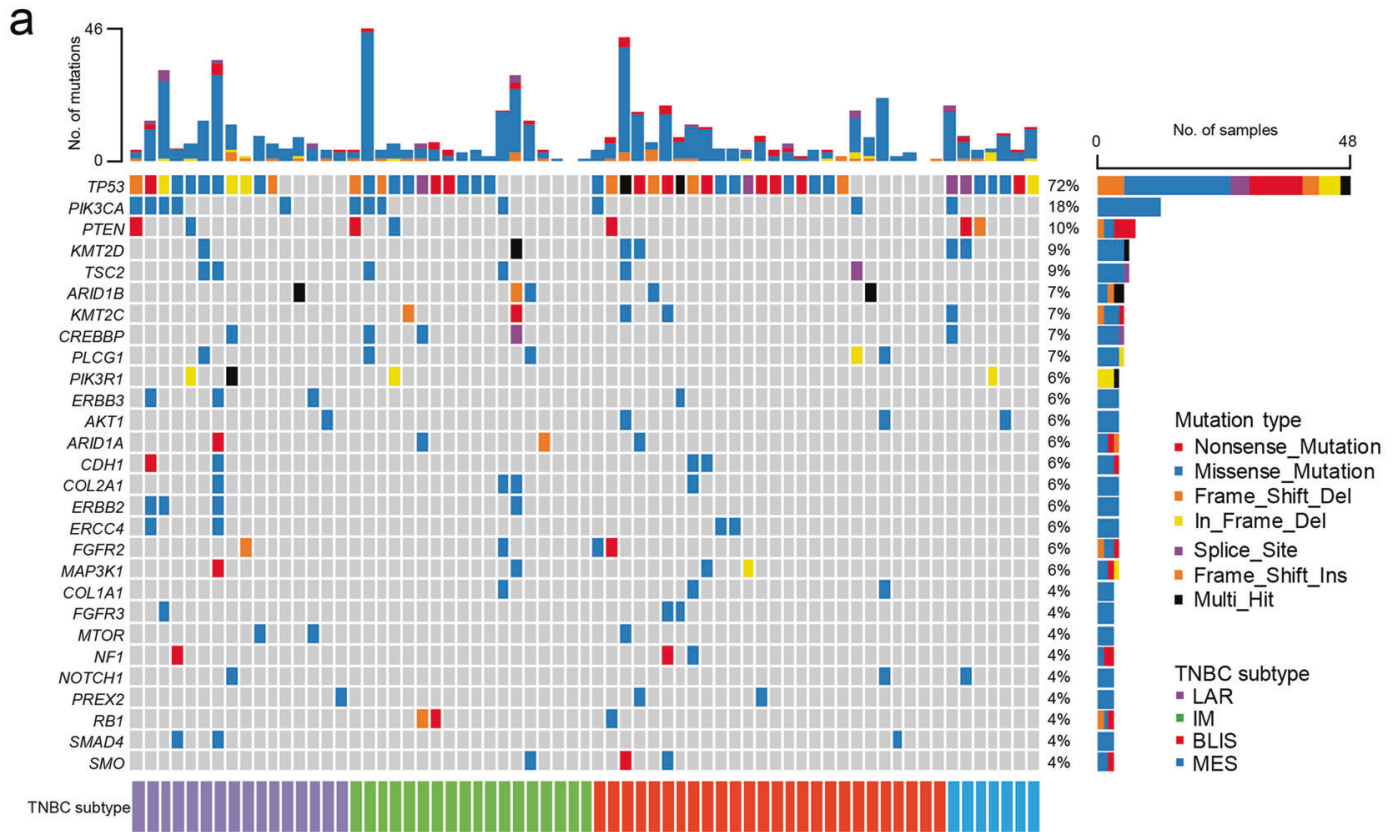

b

Primary vs metastasis

C

FUSCC vs MSKCC

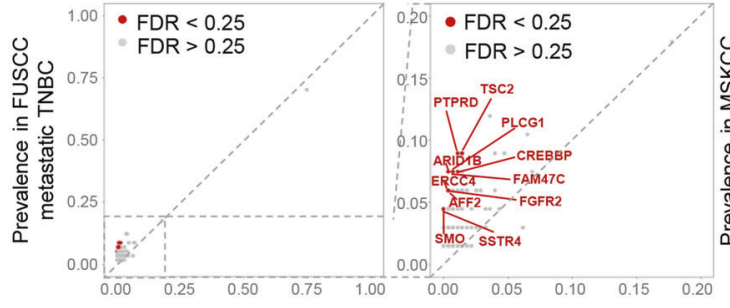

Prevalence in FUSCC early TNBC

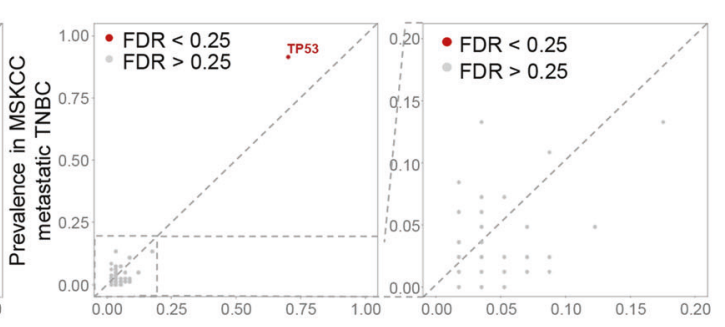

Prevalence in FUSCC metastatic TNBC

Fig. 3 Genomic landscape of refractory TNBC. a The genomic landscape of the patients in the FUTURE trial. b, c Comparison of mutation frequency between primary and metastatic TNBC (b) and between FUSCC and MSKCC metastatic TNBC (c). MSKCC, Memorial Sloan-Kettering Cancer Center; FDR, false discovery rate.

pathway. In particular, one TNBC with FGFR1 amplification benefited from anlotinib after PD from anti-AR plus anti-CDK4/6 therapy, and another patient with AR $(+)$ and CD8 (+, 25\%) benefited from anti-PD-1 therapy. These results spurs further exploration for therapeutic targets in arm B (Supplementary information, Fig. S6). In addition, arm D tested the hypothesis that the BLIS subtype TNBCs with germline BRCA1/2 mutation may benefit from PARP inhibitors, but all of the three assessable patients in arm $D$ had progressed at first evaluation (Supplementary information, Fig. S7). We speculate that these inconsistencies may have been due to the small sample size, and three subjects in this arm were platinum-refractory patients. ${ }^{38,39}$ A larger sample size may yield different results, while in the meantime, we need to continue searching for more optimal targets for tumors of this subtype.

We also compared the mutational landscape between the primary and metastatic TNBCs. Some low-frequent mutated genes, such as PTPRD, TSC2, PLCG1, ARID1B, CREBBP and FAM47C were enriched in metastatic samples. PTPRD mutation was previously reported in the TCGA cohort, ${ }^{40}$ both in vitro and in vivo experiments confirmed that PTPRD acted as a negative regulator of breast cancer metastasis, possibly via downstream IL-6/STAT3 cascade and E2F regulation. ${ }^{41,42}$ TSC2 was a putative tumor suppressor gene in the $\mathrm{PI} 3 \mathrm{~K} / \mathrm{AKT} / \mathrm{mTOR}$ pathway and its mutation was also observed in $\mathrm{HR}^{+} / \mathrm{HER} 2^{-}$metastatic breast cancers in a multi-center cohort. ${ }^{43}$ However, it was also revealed to play a protumorigenic role in breast cancer. High levels of TSC2 were found to be correlated with increased metastasis in breast cancer patients. ${ }^{44}$ Phosphorylation of TSC2 was also illustrated to activate the MTORC1 pathway and thus mediated drug resistance toward several targeted therapies of $\mathrm{HR}^{+}$breast cancer. ${ }^{45-47}$ The relationship between PLCG1 and breast cancer has yet to be studied in depth, with only one research mentioning PLCG1 expression as a predictor for the $A K T$ inhibitor response in vitro. ${ }^{48}$ ARID1B was a paralog of frequently mutated tumor suppressor gene ARID1A. $A R I D 1 B$ overexpression was found to be associated with poor prognosis in TNBC patients, but the mechanism awaits further exploration. ${ }^{49,50}$ CREBBP participates in chromosomal remodeling similar to $A R I D 1 A / B$ and was identified as a binding protein of CREB. While the relevance between CREBBP and breast cancer metastasis has been poorly understood, the CREBBP/ $\beta$-catenin/ FOXM1 axis plays a vital role in TNBC drug resistance via elevating cancer stem cell abundance. ${ }^{51}$ FAM $47 C$ was a rarely mutated gene and its function remained elusive. In summary, the impact of these infrequently mutated and metastatic-enriched genes remains largely unknown and warrants further exploration.

Owing to the small sample size of this study, some biomarkers, such as TOP2A mutation and CD8 IHC score, need further validation. Similarly, further evidence will be required before our efficacy results may be applied upon the general population. However, the majority of targeted therapies featured in our study arms have been reported in other publications, including 
a TOP2A mutation in arm C
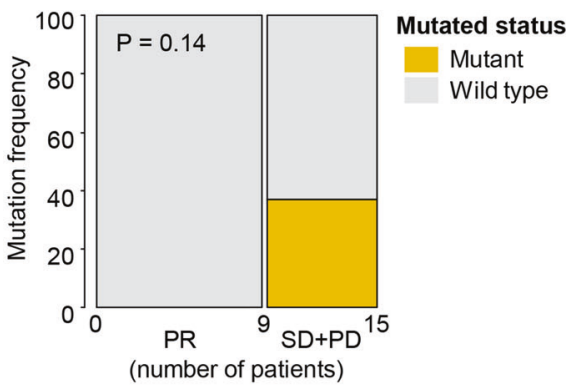

b

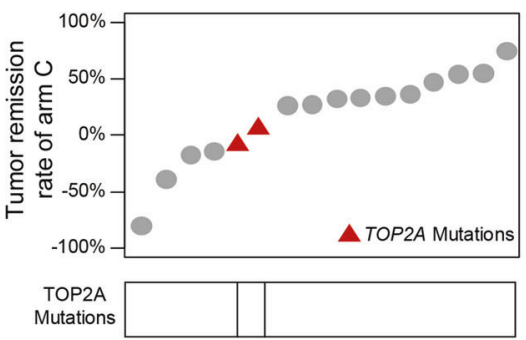

C

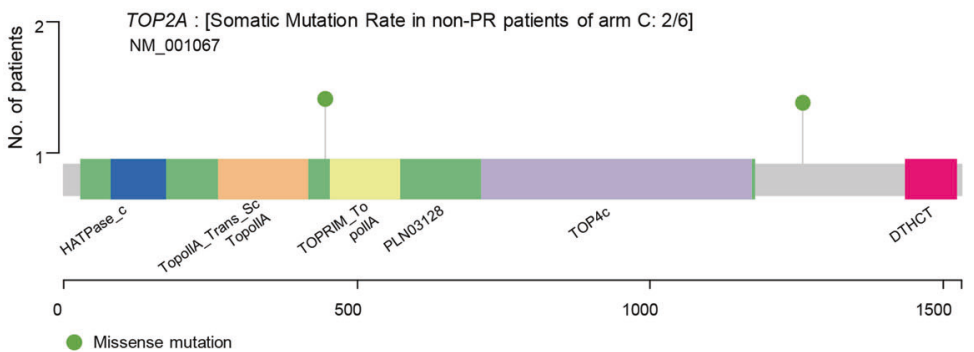

Fig. 4 Potential predictors of response for immunotherapy in IM subtype of TNBC. a TOP2A mutation in PR and non-PR patients of arm C. b Relationship between TOP2A mutation and tumor remission rate of arm C. c The change of amino acid positions related to TOP2A mutations in non-PR patients of arm C.

preliminary results from clinical studies. ${ }^{17,20,35}$ Therefore, the main purpose of this clinical trial was to prove that a combination of TNBC subtyping and genomic sequencing can help screen for patients on whom these targeted treatments would be most effective. The fact that we were able to achieve a favorable efficacy, despite out enrollment of heavily pretreated patients, suggests that our method of screening may greatly benefit the precision treatment of refractory metastatic TNBCs.

Overall, the FUTURE trial has shown that the combination of molecular subtyping and targeted sequencing was a promising treatment strategy for refractory metastatic TNBCs. Current findings of the FUTURE trial would promote further clinical research on precision treatment of TNBCs.

\section{MATERIALS AND METHODS}

\section{Study population}

We recruited patients with refractory metastatic TNBC at FUSCC from October 18th, 2018 to March 25th, 2020. Refractory metastatic TNBC was defined as metastatic TNBC patient who experienced disease progression during or following standard treatment with chemotherapy (including anthracyclines, taxanes, platinums, vinorelbine, capecitabine and gemcitabine). Eligibility criteria included: (1) female patients diagnosed with metastatic breast carcinoma with an $\mathrm{ER}^{-}, \mathrm{PR}^{-}$and HER2 phenotype. (2) central pathologic examination of tumor specimens performed by the Department of Pathology at FUSCC (ER, PR and HER2 status was independently confirmed by two experienced pathologists based on immunochemical analysis and in situ hybridization). We used $<1 \%$ positively stained cells as the cutoff for ER/PR negativity in immunohistochemistry testing according to the American Society of Clinical Oncology/ College of American Pathologists Guideline. Eligibility criteria also included having adequate performance status (ECOG grade $0-2$ ) and at least one target lesion suitable for biopsy. Uncontrolled brain metastasis was excluded from the enrollment. Details of the inclusion and exclusion criteria are provided in the study protocol in Supplementary Methods. All patients provided written informed consent. This study was approved by the FUSCC Ethics Committee.

\section{Study design and oversight}

The FUTURE trial is a phase $\mathrm{Ib} / \mathrm{Il}$, open-label, umbrella trial evaluating the efficacy and safety of multiple targeted treatments based on tumor characteristics of patients with refractory metastatic TNBC. The protocol of this study is available in Supplementary Methods.

As it took nearly one and a half months to receive the sequencing report, the screened patients were suggested to undergo biopsy to provide specimen for TNBC IHC subtyping and molecular tumor-biomarker assessment while being treated with earlier lines of therapy, or at the time of disease progression after the most recent line of standard therapy. Patients who were biopsied and were still undergoing standard chemotherapy could then be enrolled after disease progression. Their pre-obtained specimen-derived testing report would be used for arm assignment.

The samples of baseline tumor biopsy were used to conduct TNBC IHC subtype staining and an FUSCC NGS panel sequencing which detected somatic and germline mutations of 484 breast cancer-specific genes (Supplementary information, Fig. S1 and Table S1). AR, CD8 and FOXC1 were chosen as optimal IHC biomarkers for TNBC subtyping. We considered the differential expression analysis of RNA sequencing data, the correlation between the mRNA and protein expression and the feasibility for $\mathrm{IHC}$ in selecting optimal IHC biomarkers. Detailed selection steps are described in Supplementary Methods.

Patients who consented to enroll in the FUTURE trial were assigned to one of these seven treatment arms based upon their subtype and genomic biomarker. The treatment arms included: (A) pyrotinib, a HER2 receptor inhibitor, with capecitabine for the LAR subtype with ERBB2 somatic mutation, (B) androgen (AR) inhibitor (SHR3680) with CDK4/6 inhibitor (SHR6390) for the LAR subtype without ERBB2 somatic mutation, (C) anti PD-1 (SH1210) with nabpaclitaxel for the IM subtype, (D) PARP inhibitor (SH3162) included therapy for the BLIS subtype with BRCA1/2 germline mutation, $(E)$ 
anti-VEGFR (apatinib or famitinib) included therapy for the BLIS subtype without BRCA1/2 germline mutation, (F) anti-VEGFR (famitinib) included therapy for the MES subtype without PI3KAKT pathway mutation, and (G) mTOR inhibitor (everolimus) with nab-paclitaxel for the MES subtype with PI3K-AKT pathway mutation. All the treatment was continued until disease progression, patient withdrawal, or unacceptable toxic effects. Detailed drug usage and dose modifications are provided in Supplementary Methods. This study was registered with ClinicalTrials.gov, number NCT03805399.

\section{Efficacy evaluation}

Computed tomography (CT) and magnetic resonance imaging (MRI) were performed at baseline and at the second cycle after the start of treatment. Subsequent imaging was performed at one or two cycle intervals until disease progression. Assessment of response was performed according to Response Evaluation Criteria in Solid Tumors version 1.1 [RECIST v1.1]. The primary end point of the FUTURE trial was the ORR after two cycles. The secondary end point included DCR, progression-free survival (PFS), overall survival (OS) and safety. ORR was defined as the percentage of patients that experienced $C R$ or $P R$ of the disease. $D C R$ was defined as the percentage of patients that experienced CR, PR or SD. PFS was assessed from the starting date of targeted treatment therapy to the earliest sign of PD or death as a result of any cause. OS was assessed from the starting date of targeted treatment therapy to death as a result of any cause. Duration of response (DOR) was calculated as the date of the first evaluation showing documented PR or CR to the date of the first PD or death, whichever is earlier.

\section{Safety}

Safety evaluations included assessments of $A E s$ and serious adverse events (SAEs), laboratory safety evaluations, vital signs, and physical examination. AEs were assessed in accordance with the National Cancer Institute Common Terminology Criteria for Adverse Events, version 4.0 (CTCAE V4.0). For AEs with various grades, the maximum reported grade was used in Table 2.

Statistical analysis

The interim analysis was planned to be conducted when 20 subjects were enrolled in at least one arm, and at least one subject was enrolled in each arm, to preliminarily evaluate the efficacy and safety of the drug combination in each arm. With the estimated enrollment speed, around $50 \%$ of subjects would have been enrolled by the interim analysis time point. The ITT and PP populations were collected for analysis. The ITT population was defined as all enrolled population. The PP population was a subgroup of patients who were compliant with the protocol and without any major protocol violations. The efficacy of treatment was analyzed in the ITT and PP populations. The patients without at least one post-baseline efficacy evaluation were excluded from the PP population. The ORR and DCR with the $95 \% \mathrm{Cl}$ were calculated with the Clopper-Pearson method. PFS and OS with $95 \% \mathrm{Cl}$ were assessed with the Kaplan-Meier method. Median follow-up time were calculated with the reverse Kaplan-Meier method. Student's $t$-test, Wilcoxon's test, and Kruskal-Wallis test were utilized to compare continuous variables and ordered categorical variables. Pearson's chi-square test or Fisher's exact test were employed for the comparison of unordered categorical variables. All the tests were two sided, and $P<0.05$ was regarded statistically significant, unless otherwise stated. $\mathrm{R}$ version 3.6.1 (Foundation for Statistical Computing, Vienna, Austria) was used for statistical analysis.

\section{ACKNOWLEDGEMENTS}

We are grateful to the patients and their families who contributed to this study. This work was funded by Hengrui Medicine Co., Ltd (Lianyungang, China), the National
Natural Science Foundation of China (81922048, 81874112, 81874113 and 91959207), the Fok Ying-Tong Education Foundation for College Young Teachers (171034), the Training Plan of Excellent Talents in Shanghai Municipality Health System (2017YQ038), the "Chen Guang" project supported by Shanghai Municipal Education Commission, and Shanghai Education Development Foundation (17CG01), Shanghai Pujiang Program (18PJD007), the Training Plan of Excellent Talents of Fudan University Shanghai Cancer Center (YJYQ201602), the Municipal Project for Developing Emerging, and Frontier Technology in Shanghai Hospitals (SHDC12010116), the Cooperation Project of Conquering Major Diseases in Shanghai Municipality Health System (2013ZYJB0302), the Innovation Team of Ministry of Education (IRT1223), the Shanghai Key Laboratory of Breast Cancer (12DZ2260100), and the Shanghai 3-year Action Plan for Traditional Chinese Medicine (ZY[20182020]-CCCX-2005-04).

\section{AUTHOR CONTRIBUTIONS}

Z.-M.S., Z.-H.W. and Y.-Z.J. developed the concepts. Y.L. and Z.-H.W. carried out the clinical trial. Y.-Z.J., Y.L., Y.X., X.H., L.J. and J.D. collected the data of the patients. Y.-Z.J., Y.L., Y.X., X.H., L.J. and J.D. analyzed the data. Y.-Z.J., Y.L. and Y.X. wrote the original paper. Y.-Z.J., Y.L., Y.X., W.-J.Z., X.Z., J.Z., D.M., C.V., D.G.S. and V.K. reviewed and edited the paper. Y.-Z.J. and Z.-M.S. supervised the study and acquired the funding.

\section{ADDITIONAL INFORMATION}

Supplementary information accompanies this paper at https://doi.org/10.1038/ s41422-020-0375-9.

Competing interests: Xiaoyu Zhu and Jianjun Zou are employees of Jiangsu Hengrui Medicine Co, Ltd. No other potential conflicts of interest were reported.

\section{REFERENCES}

1. Waks, A. G. \& Winer, E. P. Breast cancer treatment: a review. JAMA 321, 288-300 (2019).

2. Harbeck, N. \& Gnant, M. Breast cancer. Lancet 389, 1134-1150 (2017).

3. Denkert, C., Liedtke, C., Tutt, A. \& von Minckwitz, G. Molecular alterations in triplenegative breast cancer-the road to new treatment strategies. Lancet 389, 2430-2442 (2017).

4. Bianchini, G., Balko, J. M., Mayer, I. A., Sanders, M. E. \& Gianni, L. Triple-negative breast cancer: challenges and opportunities of a heterogeneous disease. Nat. Rev. Clin. Oncol. 13, 674-690 (2016).

5. Lehmann, B. D. et al. Identification of human triple-negative breast cancer subtypes and preclinical models for selection of targeted therapies. J. Clin. Investig. 121, 2750-2767 (2011).

6. Burstein, M. D. et al. Comprehensive genomic analysis identifies novel subtypes and targets of triple-negative breast cancer. Clin. Cancer Res. 21, 1688-1698 (2015).

7. Liu, Y. R. et al. Comprehensive transcriptome analysis identifies novel molecular subtypes and subtype-specific RNAs of triple-negative breast cancer. Breast Cancer Res. 18, 33 (2016).

8. Jiang, Y. Z. et al. Genomic and transcriptomic landscape of triple-negative breast cancers: subtypes and treatment strategies. Cancer Cell 35, e425 (2019).

9. Papadimitrakopoulou, V. et al. The BATTLE-2 study: a biomarker-integrated targeted therapy study in previously treated patients with advanced non-small-cell lung cancer. J. Clin. Oncol. 34, 3638-3647 (2016).

10. Aggarwal, C. et al. SWOG S1400D (NCT02965378), a phase II study of the fibroblast growth factor receptor inhibitor AZD4547 in previously treated patients with fibroblast growth factor pathway-activated stage IV squamous cell lung cancer (lung-MAP substudy). J. Thorac. Oncol. 14, 1847-1852 (2019).

11. Edelman, M. J. et al. SWOG S1400C (NCT02154490)-a phase II study of palbociclib for previously treated cell cycle gene alteration-positive patients with stage IV squamous cell lung cancer (lung-MAP substudy). J. Thorac. Oncol. 14, 1853-1859 (2019).

12. Langer, C. J. et al. SWOG S1400B (NCT02785913), a phase II study of GDC-0032 (Taselisib) for previously treated PI3K-positive patients with stage IV squamous cell lung cancer (lung-MAP sub-study). J. Thorac. Oncol. 14, 1839-1846 (2019).

13. Herbst, R. S. et al. Lung master protocol (lung-MAP)-a biomarker-driven protocol for accelerating development of therapies for squamous cell lung cancer: SWOG S1400. Clin. Cancer Res. 21, 1514-1524 (2015).

14. Berry, S. M., Connor, J. T. \& Lewis, R. J. The platform trial: an efficient strategy for evaluating multiple treatments. JAMA 313, 1619-1620 (2015).

15. Sicklick, J. K. et al. Molecular profiling of cancer patients enables personalized combination therapy: the I-PREDICT study. Nat. Med. 25, 744-750 (2019). 
16. Rodon, J. et al. Genomic and transcriptomic profiling expands precision cancer medicine: the WINTHER trial. Nat. Med. 25, 751-758 (2019).

17. Nanda, R. et al. Pembrolizumab in patients with advanced triple-negative breast cancer: phase lb KEYNOTE-012 study. J. Clin. Oncol. 34, 2460-2467 (2016).

18. O'Shaughnessy, J. et al. Phase III study of iniparib plus gemcitabine and carboplatin versus gemcitabine and carboplatin in patients with metastatic triplenegative breast cancer. J. Clin. Oncol. 32, 3840-3847 (2014).

19. Bardia, A. et al. Sacituzumab govitecan-hziy in refractory metastatic triplenegative breast cancer. N. Engl. J. Med. 380, 741-751 (2019).

20. Traina, T. A. et al. Enzalutamide for the treatment of androgen receptorexpressing triple-negative breast cancer. J. Clin. Oncol. 36, 884-890 (2018).

21. Bardia, A. et al. Efficacy and safety of anti-trop-2 antibody drug conjugate sacituzumab govitecan (IMMU-132) in heavily pretreated patients with metastatic triple-negative breast cancer. J. Clin. Oncol. 35, 2141-2148 (2017).

22. Zehir, A. et al. Mutational landscape of metastatic cancer revealed from prospective clinical sequencing of 10,000 patients. Nat. Med. 23, 703-713 (2017).

23. Denkert, C. et al. Tumour-infiltrating lymphocytes and prognosis in different subtypes of breast cancer: a pooled analysis of 3771 patients treated with neoadjuvant therapy. Lancet Oncol. 19, 40-50 (2018).

24. Dirix, L. Y. et al. Avelumab, an anti-PD-L1 antibody, in patients with locally advanced or metastatic breast cancer: a phase $1 \mathrm{~b}$ JAVELIN solid tumor study. Breast Cancer Res. Treat. 167, 671-686 (2018).

25. Emens, L. A. et al. Long-term clinical outcomes and biomarker analyses of atezolizumab therapy for patients with metastatic triple-negative breast cancer: a phase 1 study. JAMA Oncol. 5, 74-82 (2019).

26. Adams, S. et al. Pembrolizumab monotherapy for previously treated metastatic triple-negative breast cancer: cohort A of the phase II KEYNOTE-086 study. Ann. Oncol. 30, 397-404 (2019).

27. Adams, S. et al. Pembrolizumab monotherapy for previously untreated, PD-L1positive, metastatic triple-negative breast cancer: cohort B of the phase II KEYNOTE-086 study. Ann. Oncol. 30, 405-411 (2019).

28. Tolaney, S. M. et al. Phase $1 \mathrm{~b} / 2$ study to evaluate eribulin mesylate in combination with pembrolizumab in patients with metastatic triple-negative breast cancer. Asia-Pac. J. Clin. Oncol. 14, 176-176 (2018).

29. Adams, S. et al. Phase lb trial of atezolizumab in combination with nab-paclitaxel in patients with metastatic triple negative breast cancer (mTNBC). J. Clin. Oncol. 34, 1009-1009 (2016).

30. Adams, S. et al. Atezolizumab plus nab-paclitaxel in the treatment of metastatic triple-negative breast cancer with 2-year survival follow-up: a phase $1 \mathrm{~b}$ clinical trial. JAMA Oncol. 5, 334-342 (2019).

31. Schmid, P. et al. Atezolizumab and nab-paclitaxel in advanced triple-negative breast cancer. N. Engl. J. Med. 379, 2108-2121 (2018)

32. Canela, A. et al. Topoisomerase II-induced chromosome breakage and translocation is determined by chromosome architecture and transcriptional activity. Mol. Cell 75, e258 (2019).

33. Seoane, J. A., Kirkland, J. G., Caswell-Jin, J. L., Crabtree, G. R. \& Curtis, C. Chromatin regulators mediate anthracycline sensitivity in breast cancer. Nat. Med. 25, 1721-1727 (2019).

34. Kawale, A. S. \& Povirk, L. F. Tyrosyl-DNA phosphodiesterases: rescuing the genome from the risks of relaxation. Nucleic Acids Res. 46, 520-537 (2018).

35. $\mathrm{Hu}, \mathrm{X}$. et al. Multicenter phase II study of apatinib, a novel VEGFR inhibitor in heavily pretreated patients with metastatic triple-negative breast cancer. Int. J. Cancer 135, 1961-1969 (2014).

36. Cameron, D. et al. Adjuvant bevacizumab-containing therapy in triple-negative breast cancer (BEATRICE): primary results of a randomised, phase 3 trial. Lancet Oncol. 14, 933-942 (2013).
37. Sikov, W. M. et al. Impact of the addition of carboplatin and/or bevacizumab to neoadjuvant once-per-week paclitaxel followed by dose-dense doxorubicin and cyclophosphamide on pathologic complete response rates in stage II to III triple-negative breast cancer: CALGB 40603 (Alliance). J. Clin. Oncol. 33, 13-21 (2015).

38. Fong, P. C. et al. Poly(ADP)-ribose polymerase inhibition: frequent durable responses in BRCA carrier ovarian cancer correlating with platinum-free interval. J. Clin. Oncol. 28, 2512-2519 (2010).

39. Johnson, N. et al. Stabilization of mutant BRCA1 protein confers PARP inhibitor and platinum resistance. Proc. Natl. Acad. Sci. USA 110, 17041-17046 (2013).

40. Cancer Genome Atlas, N. Comprehensive molecular portraits of human breast tumours. Nature 490, 61-70 (2012).

41. $\mathrm{Yu}, \mathrm{X}$. et al. Protein tyrosine phosphatase receptor-type delta acts as a negative regulator suppressing breast cancer. Oncotarget 8, 98798-98811 (2017).

42. Yuwanita, I., Barnes, D., Monterey, M. D., O'Reilly, S. \& Andrechek, E. R. Increased metastasis with loss of E2F2 in Myc-driven tumors. Oncotarget 6, 38210-38224 (2015).

43. Lefebvre, C. et al. Mutational profile of metastatic breast cancers: a retrospective analysis. PLoS Med. 13, e1002201 (2016).

44. Liu, H. et al. Mechanism of Akt1 inhibition of breast cancer cell invasion reveals a protumorigenic role for TSC2. Proc. Natl. Acad. Sci. USA 103, 4134-4139 (2006).

45. Citi, V. et al. Phosphorylation of AKT and ERK1/2 and mutations of PIK3CA and PTEN are predictive of breast cancer cell sensitivity to everolimus in vitro. Cancer Chemother. Pharmacol. 81, 745-754 (2018).

46. Pancholi, S. et al. Tumour kinome re-wiring governs resistance to palbociclib in oestrogen receptor positive breast cancers, highlighting new therapeutic modalities. Oncogene. 39, 4781-4797 (2020).

47. Wang, $\mathrm{H}$. et al. Feedback activation of SGK3 and AKT contributes to rapamycin resistance by reactivating $\mathrm{mTORC1/4EBP1}$ axis via TSC2 in breast cancer. Int. J. Biol. Sci. 15, 929-941 (2019).

48. Jernstrom, S. et al. Drug-screening and genomic analyses of HER2-positive breast cancer cell lines reveal predictors for treatment response. Breast Cancer (Dove Med Press) 9, 185-198 (2017).

49. Cui, Y. et al. Upregulated expression of AT-rich interactive domain-containing protein 1B predicts poor prognosis in patients with triple-negative breast cancer. Oncol. Lett. 17, 3289-3295 (2019).

50. Shao, F. et al. Clinicopathological significance of ARID1B in breast invasive ductal carcinoma. Histopathology 67, 709-718 (2015).

51. Ring, A. et al. CBP/beta-Catenin/FOXM1 is a novel therapeutic target in triple negative breast cancer. Cancers (Basel) 10, 525 (2018).

Open Access This article is licensed under a Creative Commons Attribution 4.0 International License, which permits use, sharing, adaptation, distribution and reproduction in any medium or format, as long as you give appropriate credit to the original author(s) and the source, provide a link to the Creative Commons license, and indicate if changes were made. The images or other third party material in this article are included in the article's Creative Commons license, unless indicated otherwise in a credit line to the material. If material is not included in the article's Creative Commons license and your intended use is not permitted by statutory regulation or exceeds the permitted use, you will need to obtain permission directly from the copyright holder. To view a copy of this license, visit http://creativecommons. org/licenses/by/4.0/.

c The Author(s) 2020 